\title{
Extremely low frequency electromagnetic field reduces oxidative stress during the rehabilitation of post-acute stroke patients
}

\author{
Natalia Cichońn ${ }^{1, A-D, F}$, Paulina Rzeźnicka ${ }^{1, B}$, Michał Bijak ${ }^{1, A, C, D, F}$, Elżbieta Millerer, ${ }^{2,3, A, D-F}$, \\ Sergiusz Miller, ${ }^{\mathrm{A}, \mathrm{F}}$, , Joanna Saluk ${ }^{1, A, C-F}$ \\ ${ }^{1}$ Department of General Biochemistry, Faculty of Biology and Environmental Protection, University of Lodz, Poland \\ ${ }^{2}$ Department of Physical Medicine, Medical University of Lodz, Poland \\ ${ }^{3}$ Neurorehabilitation Ward, III General Hospital in Lodz, Poland \\ ${ }^{4}$ Department of Orthodontics, Medical University of Lodz, Poland \\ A - research concept and design; $B$ - collection and/or assembly of data; $C$ - data analysis and interpretation; \\ $\mathrm{D}$ - writing the article; $\mathrm{E}$ - critical revision of the article; $\mathrm{F}$ - final approval of the article
}

\section{Address for correspondence}

Natalia Cichoń

E-mail: natalia.cichon@biol.uni.lodz.pl

Funding sources

This study was supported by the University of Lodz (grant No. 506/1136)

\section{Conflict of interest}

None declared

Received on February 17, 2017

Reviewed on March 27, 2017

Accepted on May 11, 2017

\begin{abstract}
Background. One of the therapeutic methods used in stroke rehabilitation is magnetotherapy using extremely low frequency and variable pulse shape electromagnetic field (ELF-EMF).

Objectives. The aim of our study was to investigate the effect of magnetotherapy on the condition of postacute stroke patients, as measured by plasma oxidative stress markers and clinical parameters which show the progress of rehabilitation.

Material and methods. The selected 57 post-stroke patients were divided into 2 groups, those with ELF-EMF therapy and those without. The level of oxidative stress in the plasma was estimated by typical markers: thiobarbituric acid reactive substances (TBARS), thiol groups, and carbonyl groups. The effect of ELF-EMF on the course of the patients' rehabilitation following ischemic stroke was evaluated with the use of scales of physical activity and mental state: Activities of Daily Living (ADL), Mini-Mental State Examination (MMSE) and Geriatric Depression Scale (GDS).

Results. Our comparative analysis showed that all parameters of oxidative stress are significantly reduced during rehabilitation using ELF-EMF, compared to the control group rehabilitated only by kinesiotherapy. We also recorded much higher therapeutic benefits using magnetotherapy, which revealed a significant improvement of clinimetric parameters.

Conclusions. The ELF-EMF therapy meaningfully improves the overall condition of patients through a decrease of oxidative stress markers and it significantly affects the psychophysical abilities of patients after stroke. The change in carbonyl group level correlates with the change in the degree of physical and mental disability; therefore, it could be a marker for the effectiveness of rehabilitation.
\end{abstract}

Key words: stroke, rehabilitation, oxidative stress, extremely low frequency electromagnetic field therapy

DOI

10.17219/acem/73699

Copyright

Copyright by Author(s)

This is an article distributed under the terms of the

Creative Commons Attribution Non-Commercial License

(http://creativecommons.org/licenses/by-nc-nd/4.0/) 


\section{Introduction}

A large body of evidence confirms a relationship between oxidative stress and the development of neurological diseases, including cerebrovascular disorders such as stroke. ${ }^{1}$ Epidemiological studies clearly indicate that ischemic stroke is characterized by high levels of oxidative stress biomarkers and by the insufficient activity of antioxidant defense mechanisms in the brain and peripheral tissues. The excessive neuronal production of reactive species and the accumulation of oxidative damage has been proposed as one of the major factors of brain stroke pathogenesis. ${ }^{2}$

Brain damage following stroke is irreversible, but immediate and long-term rehabilitation helps improve neurotransmission and affects the mental and physical functions of the patients. One of the therapeutic methods used in stroke rehabilitation is magnetotherapy, which uses extremely low frequency and an electromagnetic field with various pulse shape quantities (ELF-EMF). This procedure is supplementary to the treatment for physical post-stroke rehabilitation. ${ }^{3}$ Magnetotherapy is one of the physical methods characterized by non-invasiveness and a broad spectrum of applications, especially in osteoarticular diseases. Moreover, variable ELF-EMF reduces muscle tension and spasticity, increases muscle strength, and exhibits an analgesic effect. The therapeutic effect of ELF-EMF is associated with improving neurotransmission. It increases blood flow, which contributes to an increase of nervous tissue metabolism and has beneficial effects on regeneration. ${ }^{4}$

There is little data on the effect of ELF-EMF on the level of oxidative stress, which is an important destructive factor in ischemic stroke. ${ }^{5}$

Ischemic stroke is the result of the obstruction of cerebral vessels: a clot is formed and blocks an artery, which causes an interruption in the blood flow to a portion of the brain. After ischemia, the subsequent reperfusion is associated with a massive production of reactive oxygen species (ROS). An uncontrolled rise in ROS concentration leads to a series of radical reactions which increase the scope of damage to biological molecules. These highly reactive compounds readily react with lipids, proteins, carbohydrates, and nucleic acids. They induce changes in the structure and function of cell membranes, and they are responsible for the modifications of proteins, lipoproteins, enzymes, hormones, and genetic material. ${ }^{6}$ In particular, cell membranes are the main target for ROS. The highly reactive products of lipid peroxidation lead to the decomposition of polyunsaturated fatty acids and the formation of the final products, including reactive aldehydes, such as malondialdehyde (MDA). MDA is one of the most popular and reliable markers that determine oxidative stress in the clinical stage. ${ }^{7}$ Lipid peroxidation is accompanied by the inactivation of membrane enzymes and the disintegration of structural proteins. Reactive oxygen species are responsible for the oxidation of the polypeptide chain.
As a result of their oxidation, proteins undergo irreversible changes that rely on the creation of carbonyl or hydroxyl groups. These modifications lead to a fragmentation of the polypeptide chain, and an aggregation or formation of cross-links. The oxidative modifications of proteins are responsible for irreversible functional changes which are significant in both tissue damage and the impairment of the regeneration process. ${ }^{8}$

Our study aimed to investigate the ELF-EMF treatment effects on the reduction of the oxidative damage of biomolecules after a stroke. To this effect, the changes in the level of oxidative stress markers were correlated with the clinimetric parameters of the patients. The impact of a standard series of ELF-EMF treatments on protein oxidative damage was evaluated based on the level of protein carbonylation and thiol groups. Furthermore, we examined the potential antioxidative effect of magnetotherapy on plasma lipid peroxidation by measuring the level of MDA and other thiobarbituric acid reacting substances (TBARS). The effect of ELF-EMF on the course of the patients' rehabilitation following ischemic stroke was evaluated with the use of the Activities of Daily Living scale (ADL), the Mini-Mental State Examination (MMSE), and the Geriatric Depression Scale (GDS). The differences in the values of clinimetric scale parameters before and after rehabilitation reflect the effectiveness of treatments by evaluating the motor ability and cognitive ability of patients.

\section{Material and methods}

\section{Subject presentation}

Fifty-seven patients following ischemic stroke were enrolled in the study. The patients were randomly divided into 2 groups: the ELF-EMF group $(n=23)$ and the nonELF-EMF one $(n=34)$. From the ELF-EMF group, we excluded participants with metal or electronic implants. The ELF-EMF patients were exposed to ELF-EMF for $15 \mathrm{~min}$ with the following parameters: frequency $-40 \mathrm{~Hz}$, magnetic induction - $5 \mathrm{mT}$, and waveform - bipolar, rectangular. The tests on the non-ELF-EMF patients were conducted only with placebo exposure. The area of operation of ELF-EMF was the pelvic girdle. Magnetotherapy was conducted according to the accepted guidelines using a Magnetronic MF10 (EiE Elektronika i Elektromedycyna, Otwock, Poland). Both groups of patients had the same rehabilitation program consisting of aerobic exercise for $30 \mathrm{~min}$, neurophysiological methods for $60 \mathrm{~min}$, and psychological therapy for $15 \mathrm{~min}$. A total of $57 \mathrm{pa}-$ tients (study group age: $68.0 \pm 15.8$; control group age: $70.9 \pm 15.3$ ) with moderate stroke severity (study group National Institutes of Health Stroke Scale scores: $5.8 \pm 3.8$; control group scores: $6.2 \pm 2.7$ ) who agreed to participate were included in this analysis. Their clinical demographic characteristics are shown in Table 1 . The patients were 
Table 1. Clinical and demographic characteristics

\begin{tabular}{|c|c|c|c|c|}
\hline \multicolumn{2}{|c|}{ Clinical and demographic properties } & $\begin{array}{l}\text { Control } \\
n=34\end{array}$ & $\begin{array}{l}\text { Study group } \\
\qquad n=23\end{array}$ & $\mathrm{p}$-value \\
\hline \multirow{3}{*}{ Demographics } & age (mean \pm SD) & $70.9 \pm 15.3$ & $68.0 \pm 15.6$ & 0.05 \\
\hline & sex (female, \%) & 65.6 & 64.8 & 0.64 \\
\hline & living alone [\%] & 48.1 & 51.9 & 0.14 \\
\hline \multirow{4}{*}{ Vascular risk } & hypertension [\%] & 95.7 & 94.7 & 0.08 \\
\hline & diabetes [\%] & 24.8 & 21.7 & 0.36 \\
\hline & dyslipidemia [\%] & 74.9 & 67.9 & 0.14 \\
\hline & $\mathrm{BMI} \geq 30[\%]$ & 32 & 22 & 0.82 \\
\hline \multirow{3}{*}{ Concomitant medications } & antidepressants [\%] & 32 & 23 & 0.47 \\
\hline & ASA [\%] & 72 & 67 & 0.39 \\
\hline & NSAIDs [\%] & 21 & 24 & 0.72 \\
\hline \multirow{3}{*}{ Stroke characteristics } & $\begin{array}{l}\text { weeks since stroke } \\
\quad(\text { mean } \pm \text { SD) }\end{array}$ & $2.1 \pm 3.7$ & $1.8 \pm 3.5$ & 0.61 \\
\hline & NIHSS score (mean \pm SD) & $6.2 \pm 2.7$ & $5.8 \pm 3.8$ & 0.81 \\
\hline & ADL score (mean $\pm S D$ ) & $9.77 \pm 2.87$ & $8.80 \pm 2.35$ & 0.22 \\
\hline \multirow{3}{*}{ Lesion location } & anterior [\%] & 13 & 14 & 0.88 \\
\hline & posterior [\%] & 39 & 49 & 0.53 \\
\hline & intermediate [\%] & 48 & 33 & 0.12 \\
\hline \multirow{2}{*}{ Lesion side } & left [\%] & 58 & 54 & 0.75 \\
\hline & right [\%] & 42 & 46 & 0.62 \\
\hline
\end{tabular}

BMI - body mass index; ASA - acetylsalicylic acid; NSAIDs - nonsteroidal anti-inflammatory drugs; NIHSS - National Institutes of Health Stroke Scale; $\mathrm{ADL}$ - Activities of Daily Living scale.

followed-up with at Neurorehabilitation Ward III of the General Hospital in Łódź. They were undergoing neurorehabilitation for 4 weeks, and in that time they received no immunomodulators, immunostimulators, hormones, vitamins, minerals, or any other substances with antioxidative properties. Prior to the study, all the subjects had undergone medical check-ups including neurological and internist examinations. Blood samples were collected 3 times at an interval of 20 days: before treatment, after 10 , and after 20 rehabilitation treatments using magnetotherapy and/or aerobic training. All blood samples were taken in the morning (between 7 am and $9 \mathrm{am}$ ) after fasting, and stored according to the same protocol. The Ethics Committee of University of Łódź, Poland approved the protocol (No. 28/2015). All participants provided written informed consent prior to participation. Depression was screened using the Geriatric Depression Scale (GDS), which is a reliable and sensitive indicator of post-stroke depression. ${ }^{9}$ A trained psychologist researcher administered the GDS scale. The GDS, ADL, and MMSE were administered either on the same day as the blood draw or on the afternoon before.

\section{Blood sample collection}

Blood samples were collected in tubes containing Citrate Phosphate Dextrose Adenine Solution (CPDA1) and immediately centrifuged to isolate the plasma (15 min, $1500 \mathrm{~g}$ ) at $25^{\circ} \mathrm{C}$ and stored in $-32^{\circ} \mathrm{C}$ until further processing.

\section{Determination of protein carbonyl groups}

The protein carbonyl groups in the human blood plasma were detected using the ELISA method described by Alamdari et al. ${ }^{10}$ The linearity of the ELISA method was confirmed by the construction of a standard curve ranging from 0.1 to $10 \mathrm{nmol}$ carbonyl groups/mg of fibrinogen. Albumin is known to be a powerful antioxidant and its concentration in the plasma is significant. But the increase of acute phase proteins with high molecular weights, such as fibrinogen, requires important protein antioxidants to counteract the high amounts of reactive oxygen species under oxidative stress conditions. The high-molecular-weight antioxidant proteins are more suitable to prevent oxidation than albumin. ${ }^{11}$ For this reason, in our study we used fibrinogen as the standard plasma oxidation protein. Moreover, fibrinogen exhibits higher adhesion to the plate than albumin. The amount of carbonyl groups present in fibrinogen was determined spectrophotometrically as described by Levine et al. The molar extinction coefficient of 2,3-diaminophenazine (OPD with horseradish peroxidase reaction product) at a wavelength of $490 \mathrm{~nm}$ is $\varepsilon=16170 \mathrm{M}^{-1} \mathrm{~cm}^{-1}$; its specific absorption value is $\mathrm{a}=79.437 \mathrm{dm}^{3} \mathrm{~g}^{-1} \mathrm{~cm}^{-1} \cdot 12$

\section{Estimation of thiol groups}

Thiol groups in plasma proteins were determined using 5,5'-dithio-bis(2-nitro-benzoic acid) (Ellman's reagent, DTNB). ${ }^{13}$ The thiol-disulfide interchange reaction between 
DTNB and a thiol compound is the basis of this spectrophotometric assay. The reaction results in a mixture of disulphides, accompanied by the release of 5-thio-2-nitrobenzoic acid (TNB), which is colorimetrically determined. The mixture of samples with DTNB was incubated $(1 \mathrm{~h}$, $37^{\circ} \mathrm{C}$ ) and then the absorbance was measured at $412 \mathrm{~nm}$. The concentration was calculated by using the molar extinction coefficient for TNB $\left(\hat{l}=13600 \mathrm{M}^{-1} \mathrm{~cm}^{-1}\right)$ and based on a specific absorption of $\mathrm{a}=68.297 \mathrm{dm}^{3} \mathrm{~g}^{-1} \mathrm{~cm}^{-1}$.

\section{Detection of thiobarbituric acid reactive substances}

Lipid peroxidation was determined by the reaction with TBA following the method described by Placer et al. ${ }^{14}$ The level of peroxidation was calculated on the basis of the molar extinction coefficient of malondialdehyde (MDA), a reliable marker of lipid peroxidation, which is condensed with TBA $\left(\varepsilon=1.56 \times 10^{5} \mathrm{M}^{-1} \mathrm{~cm}^{-1}\right)$ and is based on a specific absorption of $\mathrm{a}=2.273 \mathrm{dm}^{3} \mathrm{~g}^{-1} \mathrm{~cm}^{-1}$ and expressed in $\mathrm{nmoles}$ of $\mathrm{MDA} / \mathrm{mL}$ of plasma.

\section{Statistical analysis}

All the experiments were performed in duplicate and results were calculated as mean values. For all the subjects, the values of experimental parameters before treatments were used as the output values (100\%). The data obtained from the same subjects after appropriate treatment were expressed as a percentage of the output value. Values obtained in this way were expressed as a mean \pm SD. All the statistical analyses were performed using the StatsDirect statistical software v. 2.7.2. The obtained results were analyzed for normality with the Shapiro-Wilk test. ${ }^{15,16}$ The significance of the differences between the values obtained for the patients before and after treatments was analyzed by the paired Student's t-test or Wilcoxon signed rank tests, depending on the normality; the significance of the differences between the ELF-EMF group and the control group, however, was analyzed using the unpaired Student's t-test or U Mann-Whitney test. To compare the clinical parameters in the ELF-EMF study group and the non-ELF-EMF control group, we assessed the changes in the GDS, ADL, and MMSE values after the appropriate treatment. These results were also analyzed for normality with the ShapiroWilk test. Depending on the result of this test, an unpaired Student's t-test or U Mann-Whitney test was used. We also performed correlation analysis between changes in both the biochemical and clinical parameters. For this analysis, we used Spearman's rank correlation and Spearman's rank correlation coefficient; moreover, we designated the probability of correlation. ${ }^{17}$ For all analyses, the level of $\mathrm{p}<0.05$ was accepted as statistically significant.

\section{Results}

In our study, we determined plasma oxidative stress parameters in acute stroke patients. It is well-estimated that oxidative stress is significantly higher in stroke patients than in healthy people (data not shown).

We observed statistically significant differences in the decreased level of carbonyl groups ( $<<0.001)$ (Fig. 1) and the increased level of thiol groups ( $<<0.01)$ (Fig. 2) in plasma proteins of the patients who were rehabilitated using magnetotherapy. The reduction of oxidative stress markers was significantly greater with an increasing number of treatments (Fig. 1, 2). The antioxidant effect of rehabilitation in the non-ELF-EMF control group undergoing only aerobic exercise was clearly weaker and not statistically significant (Fig. 1, 2). Furthermore, our comparative analysis showed that all the measured parameters of oxidative stress are significantly reduced during rehabilitation with the use of both ELF-EMF and aerobic exercise, compared to the control group rehabilitated only by kinesiotherapy. We observed that the level of protein carbonylation was lower in the ELF-EMF group than in the non-ELF-EMF group, both after 10 treatments $(18 \%$ vs $7 \%$; $<$ < 0.05$)$ and after 20 sessions (36\% vs 1\%; p < 0.001). Similarly, in the study group, the level of thiol groups increased more

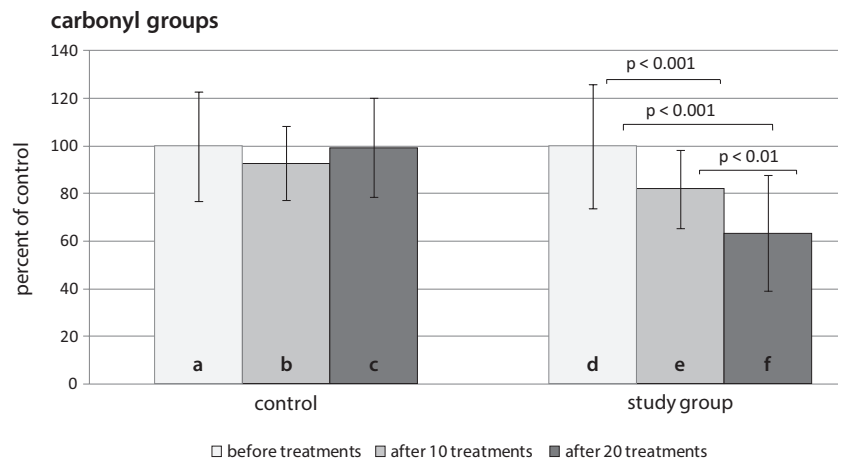

Fig. 1. The comparison of carbonyl group levels in plasma proteins obtained from the ELF-EMF group vs the non-ELF-EMF group. Statistical significance between the ELF-EMF and the non-ELF-EMF groups: $b$ vs $e=p<0.05 ; c$ vs $f=p<0.001$

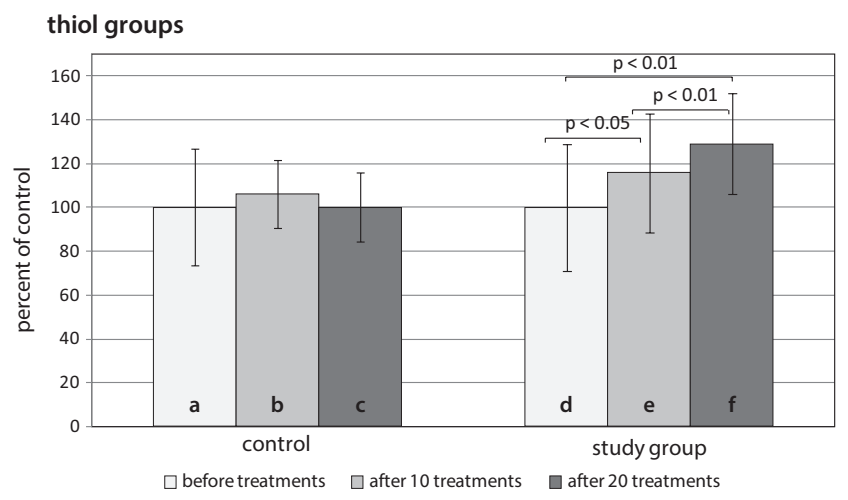

Fig. 2. The comparison of thiol group levels in plasma proteins obtained from the ELF-EMF group vs the non-ELF-EMF group. Statistical significance between the ELF-EMF and the non-ELF-EMF groups: $b$ vs $e=p<0.05 ;$ c vs $f=p<0.01$ 


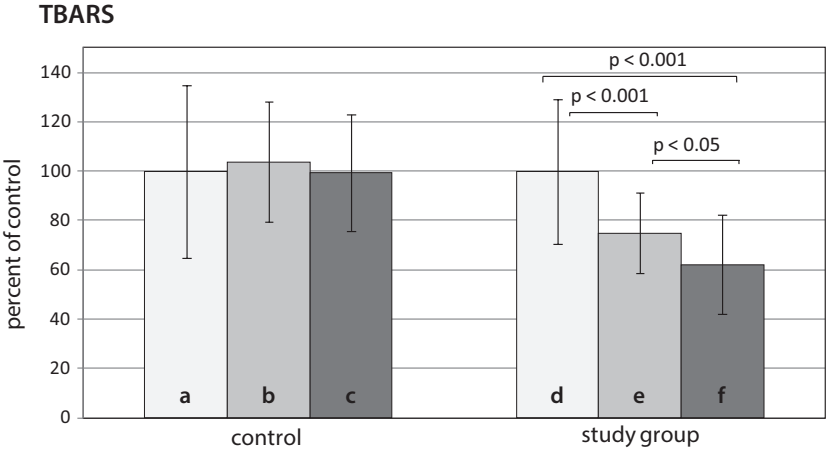

$\square$ before treatments $\square$ after 10 treatments $\square$ after 20 treatments

Fig. 3. The comparison of TBARS concentrations in plasma obtained from the ELF-EMF group vs the non-ELF-EMF group. Statistical significance between the ELF-EMF and the non-ELF-EMF groups: $b$ vs $e=p<0.001$; c vs $f=p<0.001$

than in the control group: after 10 treatments (16\% vs $6 \%$; $\mathrm{p}<0.05)$ and after 20 (29\% vs 0\%; p < 0.01).

Next, we determined the effect of magnetotherapy on lipid oxidative damage. The amount of TBARS produced in the plasma of patients after ELF-EMF therapy was significantly lower when compared to that in the plasma of patients without ELF-EMF treatments, both after 10 sessions $(p<0.001)$ and $20(p<0.001)$. The level of TBARS decreased significantly (up to $30 \%$ ) with an increasing number of rehabilitation sessions in the ELF-EMF group, but did not change significantly in the non-ELF-EMF group (Fig. 3).

In addition, we evaluated the impact of rehabilitation methods on the clinical effects, expressed in such clinimetric scales as the ADL, the MMSE, and the GDS. The ADL value in patients treated with ELF-EMF increased by about $25 \%$ compared with the control group $(\mathrm{p}<0.01)$. For the MMSE, the increase in parameters between the ELF-EMF group and the non-ELF-EMF group reached about 35\% ( $<<0.05$ ); the level of depression determined by the GDS was significantly lower in the group with magnetotherapy than in the group without it (the difference was up to 65\%) $(\mathrm{p}<0.001)$ (Table 2).

The distribution of the results of the ADL, the MMSE, and the GDS deviated from the norm in the ELF-EMF group, so the method of Spearman correlation was used for analysis. The obtained correlation parameters (Table 3) indicated a significant positive correlation between thiol group concentration and ADL value. On the other hand, we observed a significant negative correlation between carbonyl group level and ADL value. Table 3 shows the statistically significant negative correlation between carbonyl group level and the MMSE score. Moreover, the correlation parameters indicated a significant positive correlation between carbonyl group level and GDS value. The detailed course of carbonyl group correlations are shown in Fig. 4.

\section{Discussion}

It has been observed that during a stroke there is an increase in ROS production. Ischemia of brain tissue leads to depletion of the chemicals necessary for ATP synthesis, including glucose and oxygen. ${ }^{18}$ This results in an inhibition of the activity of the membrane's sodium-potassium pump, membrane depolarization, and an influx of $\mathrm{Ca}^{2+}$ into the cell. ${ }^{19}$ Accumulated cellular calcium leads to the

Table 2. The clinical parameters of ADL, MMSE, and GDS measured in the ELF-EMF group and the non-ELF-EMF group. Data are presented as the delta of the clinimetric scale before and after the standard series of treatments ( $\triangle A D L$ - the increase of ADL; $\triangle M M S E-$ the increase of MMSE; and $\triangle G D S$ - the decrease of GDS)

\begin{tabular}{|c|c|c|c|c|c|c|}
\hline \multirow{2}{*}{ Clinical scale } & \multicolumn{3}{|c|}{$\begin{array}{c}\text { non-ELF-EMF group } \\
n=34\end{array}$} & \multicolumn{3}{|c|}{$\begin{array}{l}\text { ELF-EMF group } \\
\qquad n=23\end{array}$} \\
\hline & before treatment & after treatment & $\Delta$ & before treatment & after treatment & $\Delta$ \\
\hline ADL & 9.77 & 14.73 & 4.97 & 8.80 & 15.35 & 6.55 \\
\hline MMSE & 22.28 & 25.41 & 3.08 & 20.94 & 25.61 & 4.67 \\
\hline GDS & 12.41 & 9.37 & 3.04 & 17.43 & 9.57 & 7.86 \\
\hline
\end{tabular}

ELF-EMF - extremely low frequency and variable pulse shape electromagnetic field; ADL - Activities of Daily Living scale; MMSE - Mini-Mental State Examination; GDS - Geriatric Depression Scale.

Table 3. Correlation coefficient values obtained in the ELF-EMF group for oxidative stress markers and the parameters of clinimetric scales (the ADL, the MMSE, and the GDS)

\begin{tabular}{|c|c|c|c|c|c|c|c|c|}
\hline \multicolumn{3}{|c|}{ Carbonyl groups } & \multicolumn{3}{|c|}{ Thiol groups } & \multicolumn{3}{|c|}{ TBARS } \\
\hline $\mathrm{ADL}$ & MMSE & GDS & $\mathrm{ADL}$ & MMSE & GDS & ADL & MMSE & GDS \\
\hline Rho $=-0.683$ & Rho $=-0.678$ & Rho $=0.613$ & Rho $=0.506$ & Rho $=0.392$ & Rho $=0.041$ & Rho $=-0.353$ & Rho $=-0.381$ & Rho $=-0.189$ \\
\hline$p=0.001$ & $p=0.002$ & $p=0.007$ & $p=0.023$ & $p=0.392$ & $p=0.433$ & $p=0.090$ & $p=0.054$ & $p=0.512$ \\
\hline $\begin{array}{l}\text { H1: negative } \\
\text { correlation }\end{array}$ & $\begin{array}{l}\text { H1: negative } \\
\text { correlation }\end{array}$ & $\begin{array}{l}\text { H1: positive } \\
\text { correlation }\end{array}$ & $\begin{array}{l}\text { H1: positive } \\
\text { correlation }\end{array}$ & $\begin{array}{c}\text { Ho: no } \\
\text { correlation }\end{array}$ & $\begin{array}{c}\text { HO: no } \\
\text { correlation }\end{array}$ & $\begin{array}{c}\text { HO: no } \\
\text { correlation }\end{array}$ & $\begin{array}{c}\text { HO: no } \\
\text { correlation }\end{array}$ & $\begin{array}{c}\text { HO: no } \\
\text { correlation }\end{array}$ \\
\hline
\end{tabular}

Rho - Spearman's rank correlation coefficient; $\mathrm{P}$ - probability for correlation; $\mathrm{H1}$ - hypothesis verification; ADL - Activities of Daily Living scale; MMSE - Mini-Mental State Examination; GDS - Geriatric Depression Scale. 

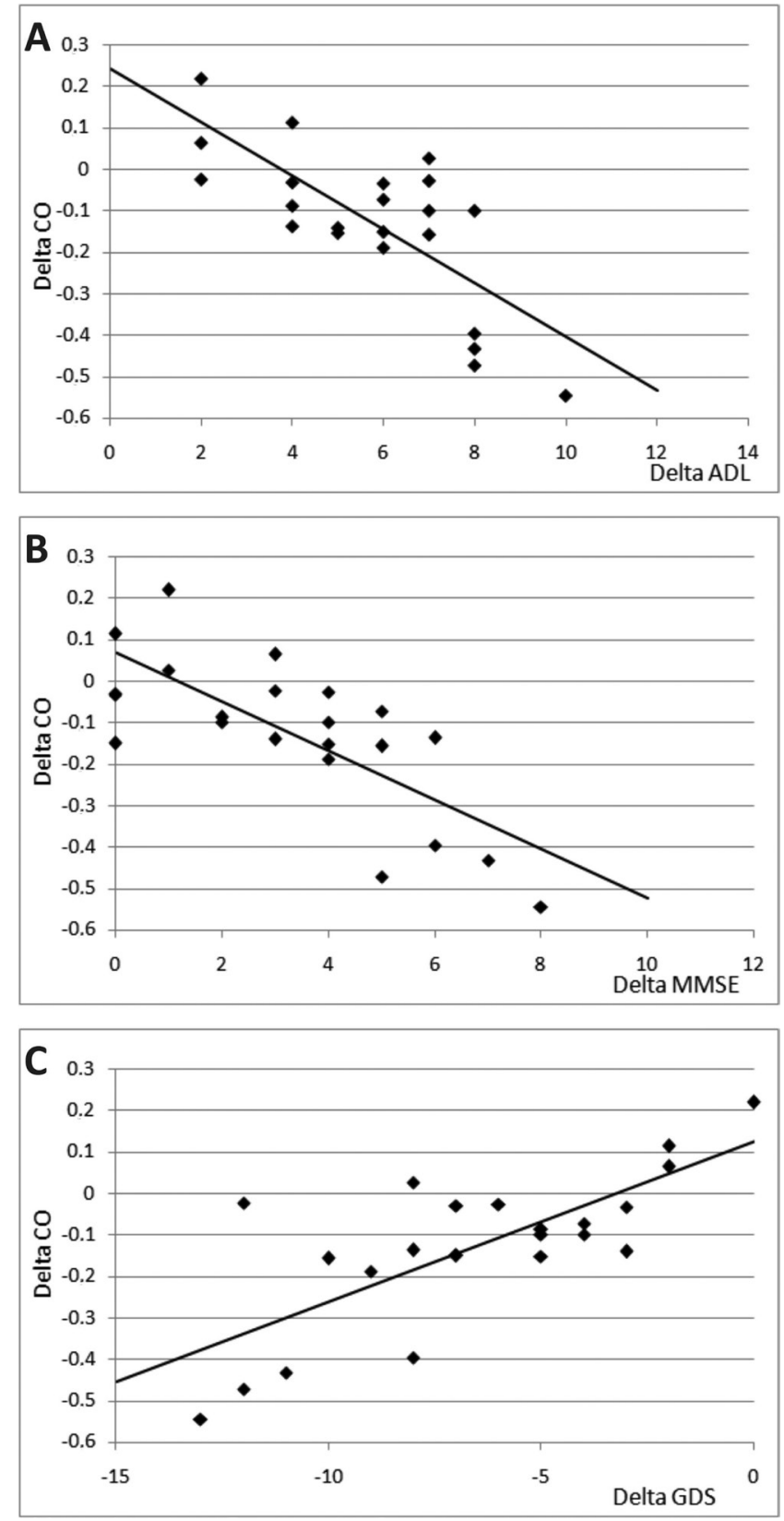

Fig. 4. Regression plots for the delta of carbonyl group levels in the ELF-EMF group (the changes of concentrations before and after a standard series of treatments) and the delta of clinical parameters established on the basis of clinimetric scales read before and after therapy. A - carbonyl group levels and $\triangle \mathrm{ADL}$; $\mathrm{B}$ - carbonyl group levels and $\triangle \mathrm{MMSE} ; \mathrm{C}$ - carbonyl group levels and $\triangle \mathrm{GDS}$

activation of many $\mathrm{Ca}^{2+}$-dependent enzymes (including proteases, nucleases, and NO synthase). This process is the basic mechanism of cell damage. On the other hand, it is the phospholipase A2 and cyclooxygenase that generate the production of free radicals. ${ }^{20}$

During hypoxia/reperfusion, ROS are generated in the reaction of xanthine oxidase, which catalyzes the oxidation of hypoxanthine and xanthine to uric acid, leading to the generation of enzymes of superoxide anions and, consequently, hydrogen peroxide and the highly toxic hydroxyl radical. ${ }^{21}$ Moreover, the mitochondrial electron chain associated with reductase and NADH cytochrome b5 plays an important role in the production of ROS. The auto-oxidation of adrenaline, noradrenaline, and thiol compounds also leads to superoxide anion generation. ${ }^{22}$

The high metabolic and oxygen activity of the brain is particularly dangerous for proteins. ROS damage strategic amino acids, thus modifying the properties of proteins. What may occur under the action of ROS is oxidation of the polypeptide chain, as well as alteration of amino acid residues. This variation could lead to a fragmentation of the polypeptide, a formation of cross-linking, and changes in the structure of the amino acids, causing a loss of the most common biological function of the protein; it may also fulfill a regulatory function. ${ }^{23}$

Ischemic stroke is one of the diseases which are directly life-threatening. It is caused by an obstruction of the brain or carotid arteries with embolic or thrombotic etiology, which leads to deficits in neurological function. The disablement and quality of life of patients is associated with motor disabilities. The neuromuscular dysfunctions responsible for such disabilities include apraxia, pain syndrome, limb spasticity, and urinary incontinence. ${ }^{24}$ The cognitive problems causing disabilities include memory loss, speech impairment, poor problem-solving skills, and disorganized thoughts. Furthermore, a stroke is often accompanied by psychosomatic complications such as anxiety, depression, emotional instability, and fatigue. ${ }^{25}$

All post-stroke patients should receive rehabilitation at an early stage of the disease. Active rehabilitation should be initiated immediately after the patient is stabilized. Although irreversible damage to the neurons occurs during a stroke, rehabilitation must be performed in order to restore the patient's fitness and return him or her to their pre-illness functional state or to adapt and achieve an optimal level of independence. The rehabilitation should deliver an improvement in practical activities and should include an improvement in cognitive impairment and changes in behavior. A preferred aspect of the rehabilitation process can be the inclusion of physical treatments such as magnetotherapy. ${ }^{3}$ The application of extremely low frequency ELF-EMF is widely used in rehabilitation primarily because of its extensive therapeutic possibilities. There are well-documented applications of ELF-EMF in rehabilitation, which reduces muscle tension and spasticity, increases muscle strength, and demonstrates an analgesic effect. The therapeutic influence of ELF-EMF is associated with its effect on nerve transduction. ELF-EMF increases blood flow, which improves the metabolism of the nervous tissue and its regeneration. In many medical centers, the use of ELF-EMF remains at the experimental stage and is not a routine method for post-stroke treatment. However, there are reports that acknowledge the use of this method as a physical treatment in improving the condition of stroke patients. ${ }^{26}$ In the literature, there are a few studies evaluating the effect of ELF-EMF on the redox system. ${ }^{27,28}$ Kurzeja et al. 
found a positive effect of ELF-EMF on the functioning of connective tissue in mice. They noted a decrease in the level of oxidative stress markers, with a simultaneous normalization of antioxidant enzyme activities. ${ }^{27}$ Similarly, Sosnowski et al. showed a reduction in hydrogen peroxide concentration after the exposure of laboratory animals to ELF-EMF, as well as a normalization of antioxidant enzyme activities. ${ }^{28}$ However, there are no data describing the in vivo ability of ELF-EMF to change the redox status of rehabilitated patients.

The aim of our study was to monitor the parameters of oxidative stress as well as the parameters assessing the patients' physical and mental improvement during rehabilitation with ELF-EMF. This study was designed to investigate the impact of ELF-EMF in comparison with classical rehabilitation using only kinesiotherapy.

The most common free radical process in the cell is a chain lipid peroxidation involving the oxidation of polyunsaturated fatty acid residues, which are part of membrane phospholipids and lipoproteins. ${ }^{29}$ Peroxidation products undergo further transformations into fragments of different lengths.

The end products of the degradation of polyunsaturated fatty acids react with other lipids, thiol groups, amino groups of proteins, and nitrogenous bases of nucleic acids causing various effects, such as a change of antigenic protein properties, enzyme inactivation, and the inhibition of replication and transcription. ${ }^{30}$

The human brain is composed of more than $60 \%$ lipids, and is particularly rich in membrane phospholipids with unsaturated fatty acid residues. Especially arachidonic acid and docosahexaenoic acid (DHA), which are the main source of polyunsaturated fatty acids in the brain, readily undergo peroxidation. In addition, the concentration of iron in some brain structures also accelerates the oxidation of lipids. The aldehyde products of lipid peroxidation may be covalently bound to a Michael reaction with the sulfhydryl groups of proteins or glutathione, reducing their concentration in the cell. ${ }^{31}$ The effect of ELF-EMF on the level of oxidative stress in vivo was examined by Rauš Balind et al. ${ }^{32}$ They noted positive consequences of applying ELF-EMF in gerbils with induced cerebral ischemia. In this study, the index of lipid peroxidation was examined in the forebrain cortex, striatum, and hippocampus. The results indicate a decrease of oxidative stress induced by global cerebral ischemia, and thereby a beneficial effect of ELF-EMF through the reduction of negative consequences which free radical species could have in the brain. ${ }^{33}$

In our study, we investigated the change of lipid peroxidation level in the plasma of post stroke patients, after the application of ELF-EMF. Because plasma is a less invasive diagnostic material, we were able to monitor the levels of markers of oxidative stress in patients during stroke rehabilitation. The results presented here indicate that the level of plasma lipid peroxidation significantly decreases with an increasing number of magnetotherapy treatments and is over $40 \%$ lower than in patients rehabilitated by exercise alone (Fig. 4).

In the present study, it was also first demonstrated that oxidative damage of plasma proteins in patients after post-stroke rehabilitation was inhibited, as evidenced by a reduction in the level of carbonyl groups (Fig. 2) and an increase in thiol group concentration (Fig. 3). The antioxidant effects of ELF-EMF used in stroke rehabilitation can provide extremely important benefits due to major implications caused by ROS in the activity of regulatory proteins, which are especially dangerous in the metabolism of neurons. Highly reactive oxygen species impair the functioning of cascades of intracellular signals and modify responses to oxidative stress gene transcription factors by changing the conformation and/or oxidation of strategic amino acids. Proteins which are particularly sensitive to oxidative stress are thioredoxin and glutathione S-transferase. ${ }^{34,35}$ The damaged oxidative proteins in general lose their biological activity; they may form aggregates because they do not undergo ubiquitination, so they are not recognized by the proteasome. ${ }^{36}$

In our previous studies, we showed that the potential ELF-EMF antioxidant mechanism is associated with a rise in the activity of the antioxidant enzyme. The ELF-EMF increases SOD and CAT activities and improves the effectiveness of post-stroke rehabilitation. Elevated SOD and CAT activities after surgery correlate with improved degrees of physical and mental disability based on the clinical scales used in research (the ADL, the MMSE, and the GDS). ${ }^{37}$

A high level of oxidative stress is the cause of complications after stroke and, according to our research as well, it significantly affects the progress of the patient's rehabilitation process. ${ }^{36,37}$ In our study, we determined the correlation between the level of markers of protein damage and the degree of clinical effects in patients rehabilitated by EMF. In order to define clinical effects, we used clinimetric scales: the ADL, the MMSE, and the GDS. The ADL is a scale which indicates the patient's independence during basic and complex activities of daily living as well as his or her gait efficiency. ADL allows for a reliable assessment of the patient's situation, his or her nursing requirements and the need to adapt the environment according to the degree of his or her impairment. ${ }^{38}$ We noted a positive correlation between increased levels of thiol groups and $\triangle \mathrm{ADL}$ (Table 3) and a negative correlation between the reduction of carbonyl groups and $\triangle \mathrm{ADL}$ (Fig. 5, Table 3). Our results suggest that under the action of ELF-EMF, the concentration of oxidative damage markers in plasma proteins is reduced, and thereby the independence of stroke patients is improved. The MMSE is used to evaluate abnormalities in the cognitive functioning of the patient and it determines the degree of dementia. Dementia is a serious problem after a stroke, characterized by numerous disorders of higher cortical functions (memory, thinking, orientation, comprehension, and learning ability). ${ }^{39}$ We found a negative 
correlation between the changes in the level of carbonyl groups and $\triangle \mathrm{MMSE}$ using magnetotherapy (Fig. 5, Table 3). The size of the concentration gradient of carbonyl groups caused by magnetotherapy was negatively correlated with the growth of MMSE scores. This indicates that the level of oxidative protein damage is referenced not only in the physical functioning of the patients, but also in his or her cognitive functions. On the other hand, the GDS scale determines the level of post-stroke depression. It is the most common psychogenic disorder occurring after a stroke. There are some reports about the somatic cause of this disease, specifying the location of ischemia and changes in biochemical parameters, among other things. In our previous studies we identified the existence of a relationship between the level of oxidative stress and the occurrence of post-stroke depression. ${ }^{40}$ We demonstrated a positive correlation between a reduction in the level of plasma protein carbonyl groups and in the degree of depression under treatment with ELF-EMF (Fig. 4, Table 3). Our results strongly support the hypothesis that rehabilitation using ELF-EMF has a positive impact on the improvement of the psychophysical condition of post-stroke patients, which is associated with a decrease in the level of protein oxidative stress parameters in vivo.

\section{Conclusions}

We emphasize that ELF-EMF significantly decreases the parameters of oxidative stress and improves the effectiveness of stroke rehabilitation. The level of changes in the concentration of plasma protein carbonyl groups correlates with the revised degrees of physical and mental disability determined by the 3 clinimetric scales used. Therefore, the plasma level of the carbonyl groups could be a marker for non-invasive evaluation of oxidative stress in vivo in the course of stroke rehabilitation, as well as a marker of the progress of the patient's recovery.

\section{References}

1. Zundorf IC, Karnath HO, Lewald J. The effect of brain lesions on sound localization in complex acoustic environments. Brain. 2014;137: 1410-1418.

2. Zhao J, Zhang $X$, Dong $L$, et al. The many roles of statins in ischemic stroke. Curr Neuropharmacol. 2014;12:564-574.

3. Sureshkumar K, Murthy GV, Munuswamy S, et al. 'Care for Stroke', a web-based, smartphone-enabled educational intervention for management of physical disabilities following stroke: Feasibility in the Indian context. BMJ Innov. 2015;1:127-136.

4. Galace de FD, Marcondes FB, Monteiro RL, et al. Pulsed electromagnetic field and exercises in patients with shoulder impingement syndrome: A randomized, double-blind, placebo-controlled clinical trial. Arch Phys Med Rehabil. 2014;95:345-352.

5. Hall ED. The contributing role of lipid peroxidation and protein oxidation in the course of CNS injury neurodegeneration and neuroprotection: An overview. In: Kobeissy FH, ed. Source Brain Neurotrauma: Molecular, Neuropsychological, and Rehabilitation Aspects. Boca Raton, FL: CRC Press, 2015:49-60.

6. Liu J, Wang Z. Increased oxidative stress as a selective anticancer therapy. Oxid Med Cell Longev. 2015;2015:294303. doi: 10.1155/2015/ $/ 294303$
7. Metta S, Basalingappa DR, Uppala S, et al. Erythrocyte antioxidant defenses against cigarette smoking in ischemic heart disease. J Clin Diagn Res. 2015;9:8-11.

8. Costantini D, Goutte A, Barbraud C, et al. Demographic responses to oxidative stress and inflammation in the wandering albatross (Diomedea exulans). PLoS One. 2015;10(8):e0133967. https://doi. org/10.1371/journal.pone.0133967

9. Nabavi SF, Dean OM, Turner A, et al. Oxidative stress and post-stroke depression: Possible therapeutic role of polyphenols? Curr Med Chem. 2015:22:343-351.

10. Alamdari DH, Kostidou E, Paletas K, et al. High sensitivity enzymelinked immunosorbent assay (ELISA) method for measuring protein carbonyl in samples with low amounts of protein. Free Radic Biol Med. 2005;39:1362-1367.

11. Olinescu RM, Kummerow FA. Fibrinogen is an efficient antioxidant. J Nutr Biochem 2001;12:162-169.

12. Levine RL, Garland D, Oliver CN, et al. Determination of carbonyl content in oxidatively modified proteins. Methods Enzymol. 1990;186: 464-478.

13. Ellman GL. Tissue sulfhydryl groups. Arch Biochem Biophys. 1959;82: $70-77$.

14. Placer ZA, Cushman LL, Johnson BC. Estimation of product of lipid peroxidation (malonyl dialdehyde) in biochemical systems. Anal Biochem. 1966;16:359-364.

15. Bijak M, Kolodziejczyk-Czepas J, Ponczek MB, et al. Protective effects of grape seed extract against oxidative and nitrative damage of plasma proteins. Int J Biol Macromol. 2012;51:183-187.

16. Saluk J, Bijak M, Nowak P, et al. Evaluating the antioxidative activity of diselenide containing compounds in human blood. Bioorg Chem. 2013;50:26-33.

17. Hauke J, Kossowski T. Comparison of values of pearson's and spearman's correlation coefficients on the same sets of data. Quaestiones Geographicae. 2011;30:87-93.

18. Liu LF, Qin Q, Qian ZH, et al. Protective effects of melatonin on ischemia-reperfusion induced myocardial damage and hemodynamic recovery in rats. Eur Rev Med Pharmacol Sci. 2014;18:3681-3686.

19. Tano JY, Gollasch M. Calcium-activated potassium channels in ischemia reperfusion: A brief update. Front Physiol. 2014;5:381. doi: 10.3389/fphys.2014.00381

20. Yano T, Fujioka D, Saito Y. Group V secretory phospholipase A2 plays a pathogenic role in myocardial ischaemia-reperfusion injury. Cardiovasc Res. 2011;90:335-343.

21. Yamaguchi M, Okamoto K, Kusano T. The effects of xanthine oxidoreductase inhibitors on oxidative stress markers following global brain ischemia reperfusion injury in C57BL/6 mice. PLoS One. 2015;10(7):e0133980. https://doi.org/10.1371/journal.pone.0133980

22. Gutowicz M. The influence of reactive oxygen species on the central nervous system. Postepy Hig Med Dosw. 2011;65:104-113.

23. Voskou S, Aslan M, Fanis P, et al. Oxidative stress in beta-thalassaemia and sickle cell disease. Redox Biol. 2015;6:226-239.

24. Young JA, Tolentino M. Stroke evaluation and treatment. Top Stroke Rehabil. 2009;16:389-410.

25. Annoni JM, Staub F, Bruggimann L, et al. Emotional disturbances after stroke. Clin Exp Hypertens. 2006;28:243-249.

26. Bassett CA. Beneficial effects of electromagnetic fields. J Cell Biochem. 1993;51:387-393.

27. Kurzeja E, Synowiec-Wojtarowicz A, Stec M, et al. Effect of a static magnetic fields and fluoride ions on the antioxidant defense system of mice fibroblasts. Int J Mol Sci. 2013;4:15017-15028.

28. Sosnowski P, Mikrut K, Paluszak J, et al. The antioxidative enzymes activity in blood of rats exposed to long-term magnetic field. Baln Pol. 1990;41,18-24.

29. Shibata T, Shimizu K, Hirano Ket al. Adductome-based identification of biomarkers for lipid peroxidation. J Biol Chem. 2017. doi: 10.1074/ jbc.M116.762609

30. Coimbra-Costa D, Alva N, Duran M, et al. Oxidative stress and apoptosis after acute respiratory hypoxia and reoxygenation in rat brain. Redox Biol. 2017;12:216-225.

31. Ionescu N, de Freitas C, Bueno AA. Perturbations in blood phosphatidylcholine and sphingomyelin fatty acid composition in a sample population of cigarette smokers. Indian J Clin Biochem. 2013;28(4): 361-367. 
32. Rauš Balind $S$, Selakovic V, Radenovic $L$, et al. Extremely low frequency magnetic field $(50 \mathrm{~Hz}, 0.5 \mathrm{mT})$ reduces oxidative stress in the brain of gerbils submitted to global cerebral ischemia. PLoS One. 2014; 9(2):e88921. https://doi.org/10.1371/journal.pone.0088921

33. Adler V, Yin Z, Tew KD, et al. Role of redox potential and reactive oxygen species in stress signaling. Oncogene. 1999;18:6104-6111.

34. Chakraborti S, Chakraborti T. Oxidant-mediated activation of mitogen-activated protein kinases and nuclear transcription factors in the cardiovascular system: A brief overview. Cell Signal. 1998;10: 675-683.

35. Paspalj D, Nikic P, Savic M, et al. Redox status in acute ischemic stroke: Correlation with clinical outcome. Mol Cell Biochem. 2015;406:75-81.

36. Eyre $H$, Baune BT. Neuroimmunological effects of physical exercise in depression. Brain Behav Immun. 2012;26:251-266.
37. Cichon N, Bijak M, Miller E, et al. Extremely low-frequency electromagnetic field (ELF-EMF) reduces oxidative stress and improves functional and psychological status in ischemic stroke patients. Bioeletromagtetics. 2017. doi. 10.1002/bem.22055

38. Heyman N, Nili F, Shahory R, et al. Prevalence of delirium in geriatric rehabilitation in Israel and its influence on rehabilitation outcomes in patients with hip fractures. Int J Rehabil Res. 2015;38:233-237.

39. Streit $S$, Limacher A, Zeller A, et al. Detecting dementia in patients with normal neuropsychological screening by Short Smell Test and Palmo-Mental Reflex Test: An observational study. BMC Geriatr. 2015; 15:90. doi: 10.1186/s12877-015-0094-0

40. Cichon N, Bijak M, Miller E, et al. Poststroke depression as a factor adversely affecting the level of oxidative damage to plasma proteins during a brain stroke. Oxid Med Cell Longev. 2015. http://dx.doi. org/10.1155/2015/408745 
